\title{
A continuum model for coupled stress and fluid flow in discrete fracture networks
}

\author{
Quan Gan · Derek Elsworth
}

Received: 23 September 2015/Accepted: 9 December 2015/Published online: 5 January 2016

(C) The Author(s) 2016. This article is published with open access at Springerlink.com

\begin{abstract}
We present a model coupling stress and fluid flow in a discontinuous fractured mass represented as a continuum by coupling the continuum simulator TF_FLAC ${ }^{3 \mathrm{D}}$ with cell-by-cell discontinuum laws for deformation and flow. Both equivalent medium crack stiffness and permeability tensor approaches are employed to characterize pre-existing discrete fractures. The advantage of this approach is that it allows the creation of fracture networks within the reservoir without any dependence on fracture geometry or gridding. The model is validated against thermal depletion around a single stressed fracture embedded within an infinite porous medium that cuts multiple grid blocks. Comparison of the evolution of aperture against the results from other simulators confirms the veracity of the incorporated constitutive model, accommodating stress-dependent aperture under different stress states, including normal closure,
\end{abstract}

Q. Gan $(\bowtie) \cdot$ D. Elsworth

Department of Energy and Mineral Engineering, EMS

Energy Institute and G3 Center, Pennsylvania State

University, University Park, PA, USA

e-mail: gan.quan@abdn.ac.uk

Q. Gan

Department of Petroleum Geology and Geology, School of Geosciences, University of Aberdeen, Aberdeen,

Scotland, UK shear dilation, and for fracture walls out of contact under tensile loading. An induced thermal unloading effect is apparent under cold injection that yields a larger aperture and permeability than during conditions of isothermal injection. The model is applied to a discrete fracture network to follow the evolution of fracture permeability due to the influence of stress state (mean and deviatoric) and fracture orientation. Normal closure of the fracture system is the dominant mechanism where the mean stress is augmented at constant stress obliquity ratio of 0.65 -resulting in a reduction in permeability. Conversely, for varied stress obliquity $(0.65-2)$ shear deformation is the principal mechanism resulting in an increase in permeability. Fractures aligned sub-parallel to the major principal stress are near-critically stressed and have the greatest propensity to slip, dilate and increase permeability. Those normal to direction of the principal stress are compacted and reduce the permeability. These mechanisms increase the anisotropy of permeability in the rock mass. Furthermore, as the network becomes progressively more sparse, the loss of connectivity results in a reduction in permeability with zones of elevated pressure locked close to the injector-with the potential for elevated pressures and elevated levels of induced seismicity.

Keywords Coupled simulation - Discrete fracture network - Geothermal reservoir - Fracture permeability $\cdot$ Stress-dependent aperture 


\section{Introduction}

Geothermal energy is a potentially viable form of renewable energy although the development of enhanced geothermal system (EGS) as a ubiquitous source has considerable difficulties in developing an effective reservoir with sustained thermal output. One key solution in generating an effective reservoir is to stimulate using low-pressure hydraulic-shearing or elevated-pressure hydraulic-fracturing. In either case, the stimulation relies on changes in effective stresses driven by the effects of fluid pressures, thermal quenching and chemical effects, each with their characteristic times-scales. Thus incorporating the effects of coupled multi-physical processes (ThermalHydraulic-Mechanical) exerts an important control on the outcome (Taron and Elsworth 2010). Therefore an improved understanding of coupled fluid flow and geomechanical process offers the potential to engineer permeability enhancement and its longevity-and thus develop such reservoirs at-will, regardless of the geological setting (Gan and Elsworth 2014a, b). This is a crucial goal in the development of EGS resources.

The subsurface fluids flow through geological discontinuities such as faults, joints and fractures and this significantly increases the complexity of the constitutive relationship linking fluid flow and solid deformation. To better represent the discontinuities in the simulation of fractured masses, a joint element model was developed based on the finite-element method for the coupled stress and fluid flow analysis by Goodman (1968), allowing the jointed rock treated as a system of blocks and links. Following this modeling approach in discretizing the fractured mass, a coupled finite element simulator by incorporating Biot's three-dimensional theory of consolidation was developed in 1982 (Biot 1941; Noorishad et al. 1982). It presented another approach to delineate the nonlinear deformation of fractures. A coupled boundary element-finite element procedures were developed to extend the analysis of both the fluid flow and deformation in discrete fractured masses into three dimensions in 1986 (Elsworth 1986). The revised direct boundary element model is capable to discretize individual fractures and assess their interactions with the adjacent fractures. A finite element model was developed to simulate the mixed mode of induced fluid-driven fracture propagation in rock mass, and the interaction with the natural fractures in 1990 (Heuze et al 1990). Since then, there are many available simulators which are capable to simulate coupled hydraulic-mechanical-thermal influence within the deformable fractured medium (Rutqvist and Stephansson 2003; Kohl et al. 1995).

The current available models are primarily divided into equivalent continuum approach and discontinuum approach. Current discontinuum models include boundary element methods (Ghassemi and Zhang 2006; McClure and Horne 2013), distinct element methods (Fu et al. 2013; Min and Jing 2003; Pine and Cundall 1985). The discontinuum approach for fractured masses assumes that the rock is assembled from individual blocks delimited by fractures. The fractures can be represented by either explicit discrete element along fractures, or by two elements-rock blocks with interfaces between them (Zhang and Sanderson 1994). Given the fact that rocks and fractures are explicitly characterized, the discontinuum approach is able to investigate the small-scale behavior of fractured rock mass, which could be more realistic in replicating the in situ behavior. However, the discontinuum model application in large reservoir scales and long term prediction demand greater computational efficiency and time.

Conversely, the major assumption for the equivalent continuum approach is that the macroscopic behavior of fractured rock masses and their constitutive relations can be characterized by the laws of continuum mechanics. The equivalent continuum approach has the advantage of representing the fractured masses at large scale, and propose simulation results of long term response. The behaviors of fractures are implicitly included in the equivalent constitutive model and modulus parameters. The most central effort in developing equivalent continuum approach is the crack tensor theory (Oda 1986). Oda developed a set of governing equations for solving the coupled stress and fluid flow with geological discontinuities, which contains the fracture size, fracture orientation, fracture volume, and fracture aperture. The rock mass is treated as an anisotropic elastic porous medium with the corresponding elastic compliance and permeability tensors.

Recent available continuum simulator TFREACT was developed by Taron (Taron et al. 2009), which couples analysis of mass and energy transport in porous fractured media (TOUGH) and combines this with mechanical deformation [FLAC3D (Itasca 2000)] with extra constitutive models including 
permeability evolution, dual-porosity poroelastic response. The purpose in this work is to extend the analysis of fractured flow and fracture deformation into the randomly distributed fractured rocks, and provide a tractable solution to optimize the potential flow and production in spatially large fractured networks. To better represent the permeability evolution due to the influence of stress, the constitutive model about the stress-dependent permeability evolution should be extended to include the scenario, which the two fracture walls are out of contact under tensile loading. The crack tensor theory is employed to determine the mechanical properties of fractured rock masses in the tensor forms, differentiating the responses of the fluid flow and stress from both the intact rocks and fractured rock masses. The constitutive model of stress-dependent permeability evolution accommodates three different stress states, including normal closure, shear dilation, and for fracture walls out of contact under tensile loading. The accuracy of the developed simulator is assessed by addressing the interaction of fracture opening and sliding deformation in response to fluid injection inside a long single fracture. Via assessing the simulation results against other discontinuum simulators, it is proved that the developed equivalent continuum model is a feasible approach to simulate the coupled thermal-hydro-mechanical behavior in discrete fractured rock masses. A series of parameter tests were conducted on the different boundary stress conditions, fracture orientation angle, fracture density. Results about the fluid transport and fracture aperture evolution implied the behavior of permeability anisotropy and flow channeling.

\section{Constitutive model development}

To implement an equivalent continuum model accommodating the fractured mass, four constitutive relations require to be incorporated. These are the formulations for a crack tensor, a permeability tensor, a model for porosity representing the fracture volume, and a model for stress-dependent fracture aperture.

\subsection{Crack tensor}

To represent the heterogeneous distribution of components of fractured rock in the simulation, the mechanical properties of fractures are characterized in tensor form based on the crack tensor theory proposed by Oda (1986). The theory is based on two basic assumptions: (1) individual cracks are characterized as tiny flaws in an elastic continuum; and (2) the cracks are represented as twin parallel fracture walls, connected by springs in both shear and normal deformation. By predefining the fracture properties, such as position, length, orientation, aperture, and stiffness, we implement crack tensor theory as a collection of disc-shaped fractures in a 3D system, and modify the distribution of modulus corresponding to the fractured rock and intact rock in each intersected element. Here the intact rock is assumed to be isotropic, the conventional elastic compliance tensor $M_{i j k l}$ for intact rock is formulated as a function of Poisson ratio, $v$, and the Young's modulus of the intact rock, $E$, as

$M_{i j k l}=\frac{(1+v) \delta_{i k} \delta_{j l}-v \delta_{i j} \delta_{k l}}{E}$

The compliance tensor $C_{i j k l}$ for the fractures are defined as a function of fracture normal stiffness $K_{n f}$, fracture shear stiffness $K_{s f}$, fracture diameter $D$, and components of crack tensors $F_{i j}, F_{i j k l}$ respectively.

$$
\begin{aligned}
C_{i j k l}= & \sum^{\text {fracnum }}\left[\left(\frac{1}{K_{n f} D}-\frac{1}{K_{s f} D}\right) F_{i j k l} .\right. \\
& \left.+\frac{1}{4 K_{s f} D}\left(\delta_{i k} F_{j l}+\delta_{j k} F_{i l}+\delta_{i l} F_{j k}+\delta_{j l} F_{i k}\right)\right]
\end{aligned}
$$

where fracnum is the number of fractures truncated in an element block, $\delta_{i k}$ is the Kronecker's delta. The related basic components of crack tensor for each crack intersecting an element are defined $F_{i j}$ as below (Rutqvist et al. 2013),

$$
\begin{aligned}
& F_{i j}=\frac{1}{V_{e}} \frac{\pi}{4} D^{3} n_{i} n_{j} \\
& F_{i j k l}=\frac{1}{V_{e}} \frac{\pi}{4} D^{3} n_{i} n_{j} n_{k} n_{l} \\
& P_{i j}=\frac{1}{V_{e}} \frac{\pi}{4} D^{2} b^{3} n_{i} n_{j}
\end{aligned}
$$

where $F_{i j}, F_{i j k l}, P_{i j}$ are the basic crack tensors, $b$ is the aperture of the crack, $V_{e}$ is the element volume, and $n$ is the unit normal to each fracture. Therefore the formula for the total elastic compliance tensor $T_{i j k l}$ of the fractured rock can be expressed as, 
$T_{i j k l}=C_{i j k l}+M_{i j k l}$

Combining Eqs. 3-5 into Eq. 2, the equivalent fracture Young's modulus $E^{f}$ and Poisson ratio $v^{f}$ can be obtained as,

$$
\begin{aligned}
E^{f} & =\frac{1}{\frac{1}{E}+\left(\frac{1}{K_{n f}}-\frac{1}{K_{s f}}\right) \frac{1}{V_{e}} \frac{\pi}{4} D^{2} n_{1}^{4}+\frac{1}{K_{s f}} \frac{1}{V_{e}} \frac{\pi}{4} D^{2} n_{1}^{4}} \\
v^{f} & =\frac{v}{E} E^{f}-\left(\frac{1}{K_{n f}}-\frac{1}{K_{s f}}\right) \frac{E^{f}}{V_{e}} \frac{\pi}{4} D^{2} n_{1}^{2} n_{2}^{2}
\end{aligned}
$$

Given the assumption that the properties of modulus are anisotropic, the equivalent bulk modulus $K$ and shear modulus $G$ for the fractured rock mass are formulated as below,

$$
K=\frac{1}{\frac{1}{K_{\text {intact }}}+\sum^{\text {fracnum }} \frac{V_{\text {ratio }}}{b}\left[\left(\frac{1}{K_{n f}}-\frac{1}{K_{s f}}\right)\left(1-n_{2}^{4}\right)+\frac{1}{K_{s f}} n_{1}^{2}\right]}
$$

$$
G=\frac{1}{\frac{1}{G_{\text {intact }}}+\sum^{\text {fracnum }} \frac{2 V_{\text {ratio }}}{b}\left[\left(\frac{1}{K_{n f}}-\frac{1}{K_{s f}}\right)\left(n_{1}^{4}-n_{1}^{2} n_{2}^{2}\right)+\frac{1}{K_{s f}} n_{1}^{2}\right]}
$$

where $K_{\text {intact }}$ is the bulk modulus of the intact rock, $G_{\text {intact }}$ is the shear modulus of the intact rock and $V_{\text {ratio }}$ is the volumetric ratio of the truncated fracture over the element volume. The stress-dependent evolution of fracture aperture will in turn update the equivalent modulus of the fractured rock masses.

\subsection{Permeability tensor and aperture evolution}

Considering that the randomly distributed fractures may be intersected by multiple elements in the reservoir gridding, the directional fracture permeability is defined as a permeability tensor $k_{i j}$, which is able to represent the orientation of fractures and the explicit fracture volume intersecting any element block.

$$
\begin{aligned}
k_{i j} & =\sum_{\text {fracnum }} \frac{1}{12}\left(P_{k k} \delta_{i j}-P_{i j}\right) \\
& =\sum_{\text {fracnum }} \frac{1}{12}\left(\frac{V_{\text {ratio }}}{b_{\text {ini }}} b^{3} n_{k}^{2} \delta_{i j}-\frac{V_{\text {ratio }}}{b_{\text {ini }}} b^{3} n_{i} n_{j}\right)
\end{aligned}
$$

where $b_{i n i}$ is the initial aperture of fracture.
The effect of stress has a direct impact in changing the evolution of the fracture aperture, which will in turn change the compliance tensor in the simulation loop. Prior models for stress permeability coupling include hyperbolic models of aperture evolution (Bandis et al. 1983; Barton and Choubey 1977), which can describe the response of fracture aperture in normal closure under the influence of in situ stress. The functionality of the hyperbolic model is mediated by the parameters of initial fracture normal stiffness $K_{n f}^{0}$, maximum closure of the fracture aperture $d_{n \max }$, initial aperture of the fracture, $b_{i n i}$, and the effective normal stress of the fracture $\sigma_{n}^{\prime}$, which is formulated as,

$\Delta d_{n}=\frac{d_{n \max }}{1+\frac{K_{n f}^{0} d_{\max }}{\sigma_{n}^{\prime}}}$

A simplified Barton-Bandis hyperbolic model (Baghbanan and Jing 2007) is adopted to model aperture evolution by introducing a new parameterthe critical normal stress $\sigma_{n c}$, which means that the normal compliance $C_{n}$ is reduced significantly when the aperture closure approaches the maximum closure. In order to simplify the hyperbolic solution it is assumed that the ratio of maximum normal closure $d_{n \max }$ relative to initial fracture aperture $b_{i n i}$ is constant at 0.9. Given this assumption, the hyperbolic normal closure equation is transformed as,

$\sigma_{n}^{\prime}=\frac{\sigma_{n c} \Delta d_{n}}{10\left(0.9 b_{i n i}-\Delta d_{n}\right)}$

and the normal stiffness is determined by the normal stress as,

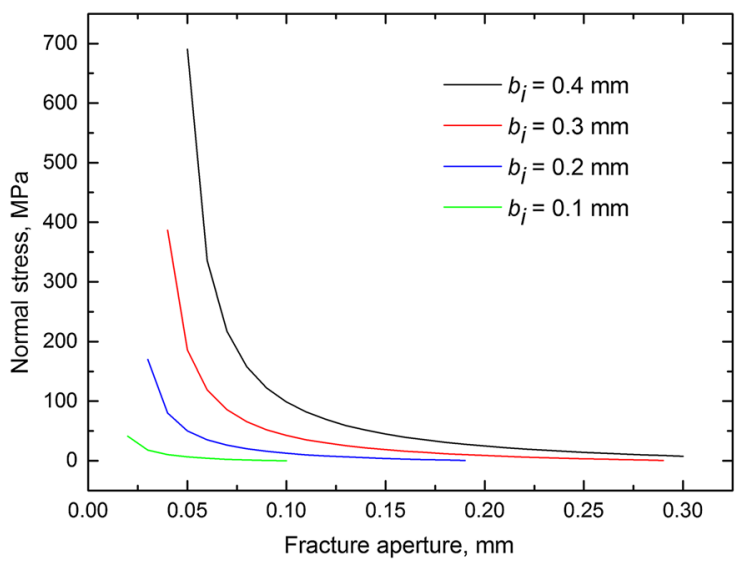

Fig. 1 The impact of initial aperture in the relationship between normal stress and fracture aperture 
$K_{n f}=\frac{\left(10 \sigma_{n}^{\prime}+\sigma_{n c}\right)^{2}}{9 \sigma_{n c} b_{i n i}}$

where $\sigma_{n c}(\mathrm{MPa})=0.487 b_{\text {ini }}(\mu \mathrm{m})+2.51$. Figure 1 shows the hyperbolic relationship between the normal stress and the aperture corresponding to different predefined apertures. This shows that the larger the initial aperture, the larger drawdown gradient of normal stress.

Fracture shear slip and related dilation are included in the simulator by lumping the influence into the response of the matrix rock being sheared. When the Coulomb failure criterion is reached, the shear displacement $u_{s}$ will generate shear dilation $b_{\text {dila }}$ in the normal direction according to the equation (Fig. 2),

$b_{\text {dila }}=u_{s} \tan \phi_{d}$

where $\phi_{d}$ is the dilation angle. Figure 2 identifies the relationship between the shear stress and displacement due to normal dilation. When the shear stress reaches the critical magnitude $\tau_{s c}$, which is determined by the Coulomb failure criterion, shear failure triggers normal dilation (red line) to increase the fracture aperture as the shear displacement increases. To incorporate the weakening response for the onset of shear failure in fractured rock masses, the reduction of a linear slope gradient (black line) represents the significant reduction of fracture shear stiffness from $K_{s 1}$ to $K_{s 2}$ due to shear failure. The magnitude of the aperture increment added by shear dilation can be calculated as,

$b_{\text {dila }}=\frac{\tau-\tau_{s c}}{K_{s}} \tan \phi_{d}$

When the fluid pressure in the fracture exceeds the normal stress across the fracture, the two walls of the

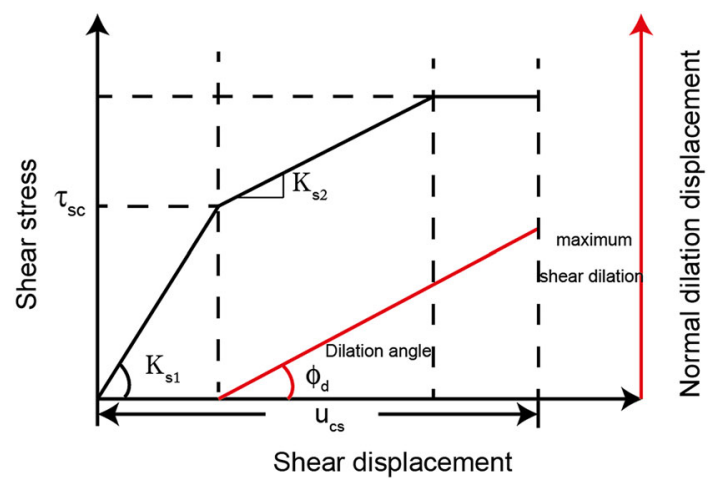

Fig. 2 Fracture normal dilation displacement evolution induced from the shear slip in fracture fracture are separated and the effective normal stress is zero (Crouch and Starfield 1991) since $P_{f}=\sigma_{n}$.

Figure 3 represents the constitutive relation for fracture aperture evolution applied in this model. The curve to the right is the regime under normal closure and shear dilation. However, as soon as the fluid pressure reaches the critical magnitude $P_{f o}$ where effective stress is zero (left side in Fig. 3), a geometrical stiffness $K_{g f}$ for a penny-shaped fracture is employed to calculate the induced normal opening displacement when the two walls are under tension. The normal opening displacement is linear with an increment of fluid pressure $\left(P_{f}-P_{f 0}\right)$. This geometrical stiffness $K_{s}^{\text {rock }}$ (Dieterich 1992), although small, is much larger than that of the fracture in shear and is defined as,

$K_{s}^{r o c k}=\eta \frac{G}{r}=\frac{7 \pi}{24} \frac{G}{r}$

where $\mathrm{G}$ is the shear modulus of the intact rock, $D$ is the fracture half length, and $\eta$ is a geometrical factor which depends on the crack geometry and assumptions related to slip on the patch (Dieterich 1992). In this study, $\eta$ is defined to represent a circular crack and is given as $\frac{7 \pi}{24}$. Therefore the equation for the fracture opening displacement $b_{\text {open }}$ is formulated as below,

$b_{\text {open }}=\frac{P_{f}-P_{f 0}}{K_{g f}}=\frac{P_{f}-P_{f 0}}{10 K_{s}^{r o c k}}=\frac{\left(P_{f}-P_{f 0}\right)}{10 \times \frac{7 \pi}{24} \frac{G}{r}}$

To summarize, Eqs. 11, 15, and 17, represent the relations for considering stress-dependent aperture

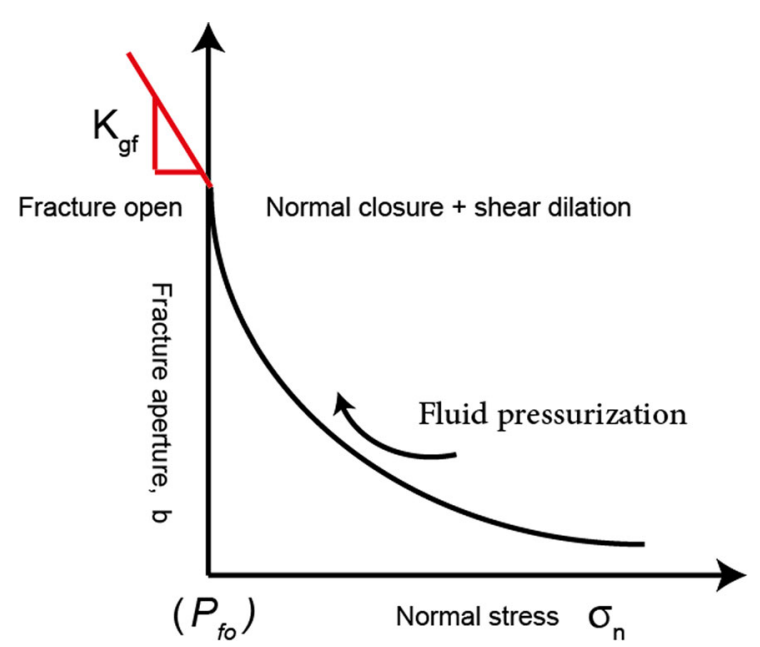

Fig. 3 Fracture aperture evolution under different normal stress state 
change including normal closure, shear dilation, and fracture opening is obtained as,

$b=b_{i n i}-\frac{9 b_{i n i} \sigma_{n}^{\prime}}{\sigma_{n c}+10 \sigma_{n}^{\prime}}+\frac{\tau-\tau_{s c}}{K_{s}} \tan \phi_{d}+\frac{\left(P_{f}-P_{f 0}\right)}{10 \times \frac{7 \pi}{24} \frac{G}{r}}$

where $b_{\text {ini }}$ is the initial aperture of the fracture, $b_{\text {normal }}$ is the reduction of aperture due to the normal closure, $\sigma_{n}^{\prime}$ is the effective normal stress of the fracture, $\sigma_{n c}$ is the critical normal stress, $\tau_{s c}$ is the critical shear stress where shear failure happens.

\subsection{Porosity model}

Considering that deformations occur independently within the two different media of fracture and matrix, it is important to accommodate the evolution of the dual porosity system with the induced strain, since the porosity is iteratively coupled in the hydro-mechanical simulations. Hence in this study, there are two different equations to represent the evolution of porosity in fracture and matrix. In terms of the matrix, the volumetric strain is employed to update the matrix porosity as (Chin et al. 2000),

$\phi_{n+1}^{m}=1-\left(1-\phi_{n}^{m}\right) e^{-\Delta \varepsilon_{v}^{s}}$

where $\Delta \varepsilon_{v}^{s}$ is the change of matrix volumetric strain, $\phi_{n+1}^{m}$ and $\phi_{n}^{m}$ are the matrix porosity at time $t_{n+1}$ and $t_{n}$ respectively. The fracture porosity is calculated as below,

Fig. 4 Equivalent continuum simulation workflow implementation in TFREACT
$\Delta \varepsilon_{v}^{f}=\frac{\Delta b}{b_{\text {ini }}}$

$\phi_{n+1}^{f}=1-\left(1-\phi_{n}^{f}\right) e^{-\Delta \varepsilon_{v}^{f}}$

where $\Delta b$ is the aperture change, $\Delta \varepsilon_{v}^{f}$ is the change of fracture volumetric strain. The assumption is made that only the aperture evolution $\Delta b$ in the normal direction is considered in calculating the fracture volumetric strain $\Delta \varepsilon_{v}^{f}$.

\subsection{Numerical simulation workflow}

Based on the constitutive model described above, the workflow for the equivalent continuum model is presented in Fig. 4. The simulation initiates with the equilibration of temperature $(T)$ and pore pressure $\left(P_{f}\right)$ in TOUGH. Subsequently, initial data describing the fracture network, including fracture orientation, trace length, aperture, and modulus are input into a FORTRAN executable. The compliance tensor transfers the composite fracture modulus with the equilibrium pore pressure distribution into FLAC3D to perform the stress-strain simulation. The revised undrained pore pressure field is then redistributed, based on principles of dual porosity poromechanics, and applied to both fracture and matrix. The stressdependent fracture aperture is calculated and updated based upon the failure state of the fracture. Since the fracture permeability and composite modulus of the fractured medium are both mediated by the magnitude

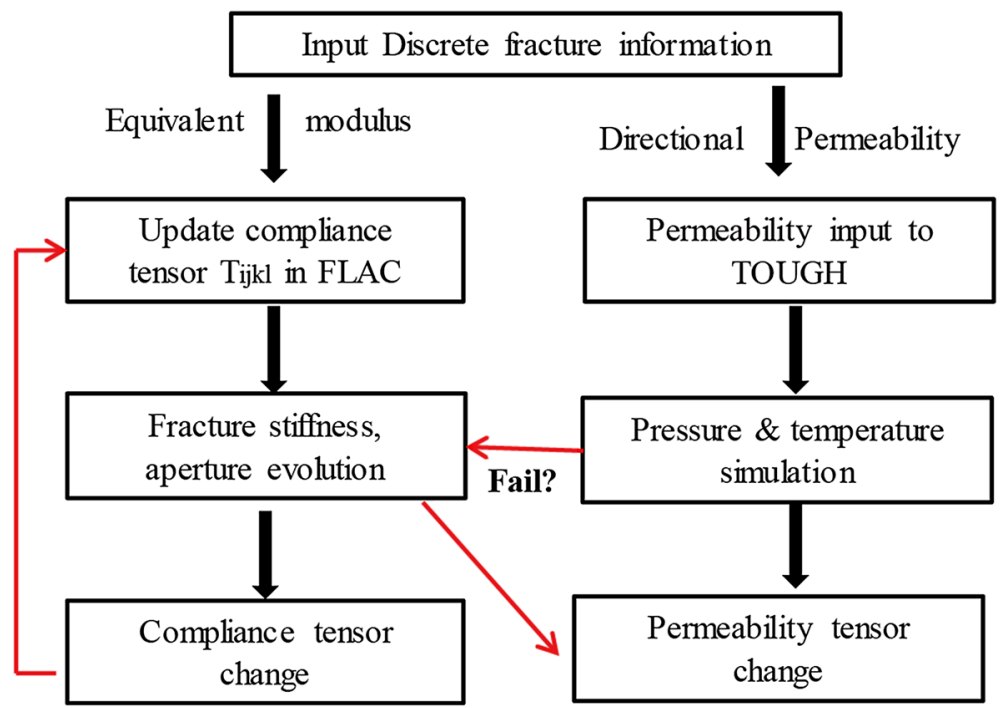


of fracture aperture, the fracture aperture is iteratively adjusted in the simulation loop to define the revised fracture permeability and to alter the compliance tensor of the composite fractured mass.

\section{Model verification}

In this section, the discrete fracture network (DFN) model is verified by comparing the results against other DFN models. The model in this study (Kelkar et al. 2015) is an injection well intersecting a single fracture that dilates and slips in response to fluid injection.

\subsection{Model setup}

Figure 5 shows the reservoir geometry applied in this study. The single fracture is oriented at $45^{\circ}$ to the principal stresses and embedded within an infinite medium. The fracture has a length of $39 \mathrm{~m}$ with an initial width of $1 \mathrm{~mm}$ and does not propagate. The injection well is centrally located along the fracture with constant injection at $6.0 \times 10^{-8} \mathrm{~m}^{3} / \mathrm{s}$. The maximum principal stress is $20 \mathrm{MPa}$ (y-direction) and the minimum principal stress is $13 \mathrm{MPa}$ (x-direction). Initial pore pressure and reservoir temperature are each uniform at $10 \mathrm{MPa}$ and $420 \mathrm{~K}$. The initial

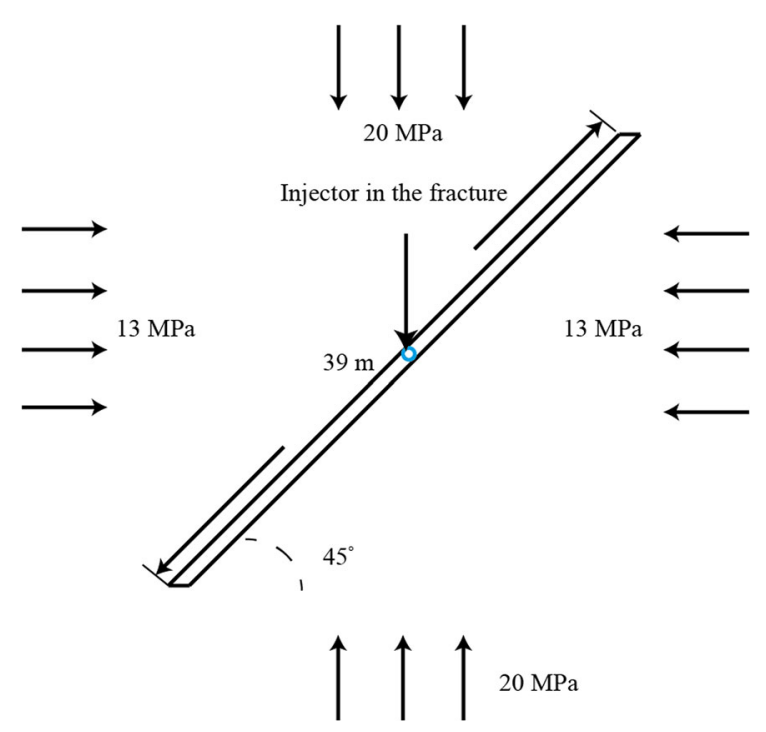

Fig. 5 Reservoir configuration for injection inside a $39 \mathrm{~m}$ long fracture within an infinite medium reservoir properties used in this model are listed in Table 1. The pre-stressed fracture is in equilibrium with the prescribed stress and pore pressure to yield the desired initial aperture of $1 \mathrm{~mm}$.

\subsection{Results discussion}

In this section, the results are compared between the contrasting conditions of isothermal injection and non-isothermal injection. To isolate the impact of the induced thermal stress effect in the aperture evolution, the liquid viscosity is set as constant for both the isothermal and non-isothermal injection conditions.

Figure 6 presents the results of fracture aperture evolution under isothermal injection conditions for the first 180 days. By comparing the results from our equivalent continuum approach against other those for discrete fracture models, including GeoFrac (Ghassemi and Zhang 2006) and CFRAC (McClure and Horne 2013) we verify that the equivalent continuum model TF_FLAC ${ }^{3 \mathrm{D}}$ is able to match results for the evolution of fracture aperture.

Figure 7 presents the evolution of injection pressure in the fracture among the three different simulators for the condition of isothermal injection. There is a good agreement between the injection pressures recovered from TF_FLAC ${ }^{3 \mathrm{D}}$ and the other discrete fracture models. In this response, the normal aperture grows slowly under initial pressurization and most rapidly as the walls lose contact and the full geometric stiffness of the fracture is mobilized as the fracture walls lose contact. The walls of the fracture are out of contact after $\sim 60$ days of injection. Due to permeability enhancement in the fracture, the injection pressure gradient in the fracture decreases after the fracture opens.

Figure 8 presents a comparison of injection pressures between isothermal and non-isothermal injection. In this, water is injected at $400 \mathrm{~K}$ for a system originally in thermal equilibrium at $420 \mathrm{~K}$. The injection pressure results for these two conditions are similar. However, there is a notable difference in the growth in fracture aperture between the two injection scenarios (Fig. 9). The major difference occurs when the fracture walls lose contact. Prior to fracture separation, the fracture aperture for non-isothermal injection is slightly larger than that for isothermal injection. This can be explained by following the evolution of the normal stress acting across the 
Table 1 Data used in the simulation (McTigue 1990)

\begin{tabular}{ll}
\hline Parameter (unit) & Magnitude \\
\hline Shear modulus $[\mathrm{G}(\mathrm{GPa})]$ & 15 \\
Poisson's ratio $(v)$ & 0.25 \\
Undrained Poisson's ratio & 0.33 \\
Matrix permeability $\left[k_{m}\left(\mathrm{~m}^{2}\right)\right]$ & $4.0 \times 10^{-19}$ \\
Matrix porosity $\left(\phi_{m}\right)$ & 0.01 \\
Biot's coefficient $(\alpha)$ & 0.44 \\
Water viscosity $[\mu(\mathrm{Pa} \mathrm{s})]$ & $3.547 \times 10^{-4}$ \\
Fluid compressibility $\left(\mathrm{MPa}{ }^{-1}\right)$ & $4.2 \times 10^{-4}$ \\
Thermal expansion coefficient of solid $\left[\alpha_{s}\left(\mathrm{~K}^{-1}\right)\right]$ & $2.4 \times 10^{-5}$ \\
Thermal diffusivity of intact porous rock $\left[c^{T}\left(\mathrm{~m}^{2} / \mathrm{s}\right)\right]$ & $1.1 \times 10^{-6}$ \\
Fluid density $\left[\rho_{w}\left(\mathrm{~kg} / \mathrm{m}^{3}\right)\right]$ & 1000 \\
Heat capacity of fluid $\left[c_{w}(\mathrm{~J} / \mathrm{kg} \mathrm{K})\right]$ & 4200 \\
Initial reservoir temperature $(K)$ & 420 \\
Injection water temperature $(K)$ & 400 \\
Initial joint normal stiffness $\left[k_{n}(\mathrm{GPa} / \mathrm{m})\right]$ & 0.5 \\
Initial joint shear stiffness $\left[k_{s}(\mathrm{GPa} / \mathrm{m})\right]$ & 50 \\
Fracture aperture initial $\left[b_{\text {ini }}(\mathrm{mm})\right]$ & 1 \\
In-situ stress $(\mathrm{MPa})$-y direction & 20 \\
In-situ stress $(\mathrm{MPa})-\mathrm{x}$ direction & 13 \\
Initial reservoir pore pressure $(\mathrm{MPa})$ & 10 \\
Injection rate $\left(\mathrm{m}^{3} / \mathrm{s}\right) / \mathrm{m}$ thickness of reservoir & $6.0 \times 10^{-8}$ \\
Friction angle, dilation angle & $30^{\circ}, 2.5^{\circ}$ \\
Fracture cohesion $[C(\mathrm{MPa})]$ & 0 \\
\hline
\end{tabular}

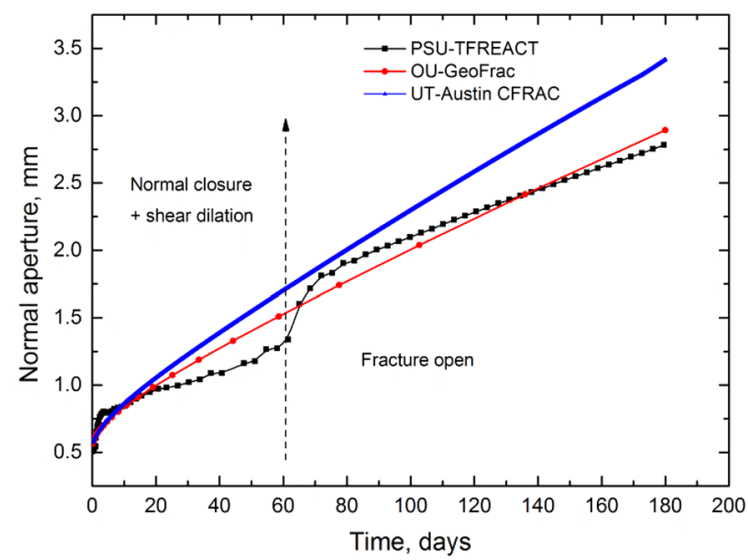

Fig. 6 Fracture aperture evolution comparisons against other discontinuum models

fracture, as noted in Fig. 10-and is a consequence of thermal unloading by the quenching fluid. The thermal unloading effect is more pronounced when the fracture is in extension (open).

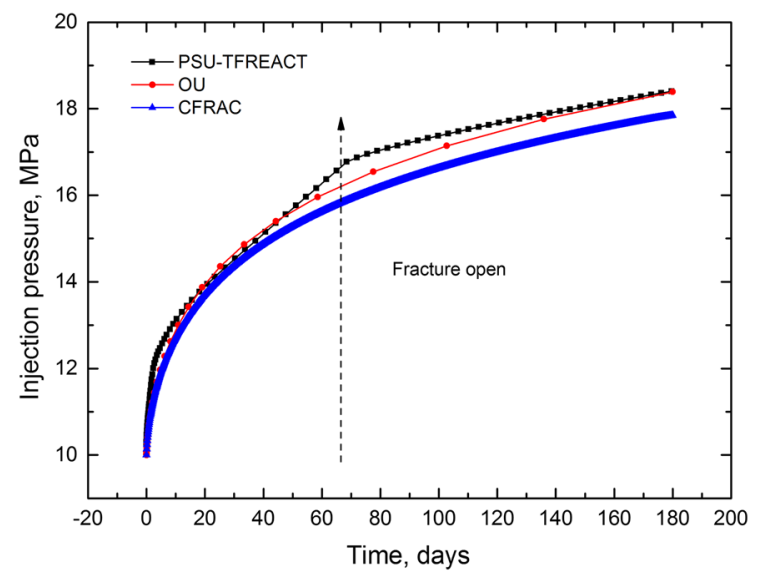

Fig. 7 Evolution of fracture injection pressures evaluated by various models

Figure 11 shows the distribution of aperture along the fracture at 72 and 180 days for both the isothermal injection and non-isothermal injection cases. There is no significant difference in the distribution of fracture 


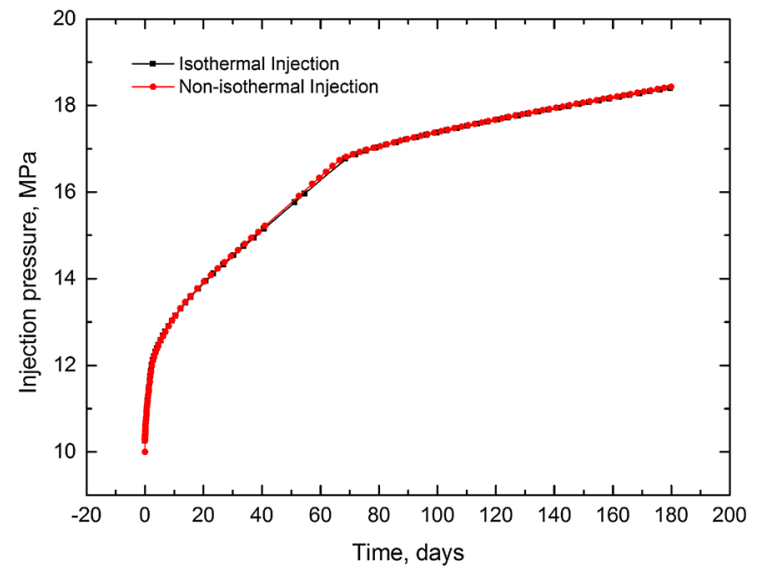

Fig. 8 Evolution of injection pressure for isothermal injection (black line) and non-isothermal injection (red line)

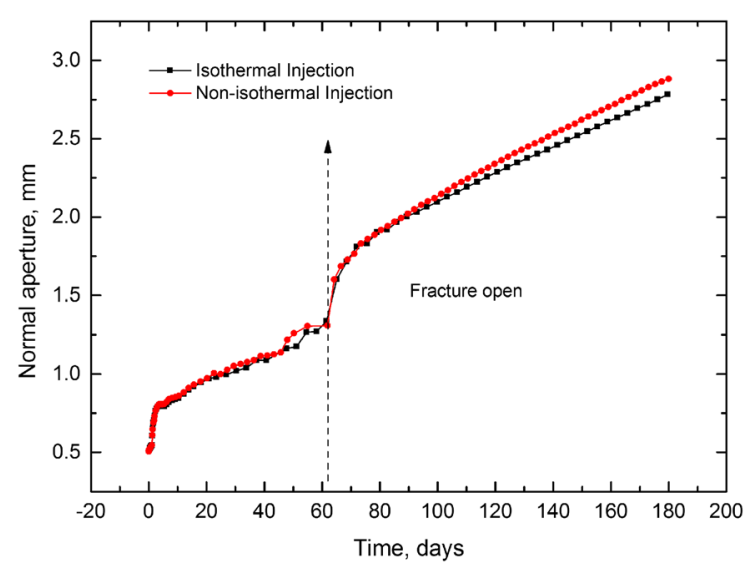

Fig. 9 Evolution of fracture normal aperture for isothermal injection (black line) and non-isothermal injection (red line), respectively

aperture for isothermal and non-isothermal cases at 72 days, although the results diverge by 180 days. By 180 days, the influence of the quenching fluid in reducing the fracture-local normal stress has become sufficiently significant to increase the aperture by $\sim 3 \%$ for a permeability increase of the order of $1.03^{3} \sim 10 \%$.

\subsection{Multi-fracture validation}

The previous TFREACT simulator is able to simulate the response of fracture aperture and pore-elasticity in the framework of orthogonal fracture sets. In this section, the simulation of multi-fracture intersection was completed between the equivalent continuum

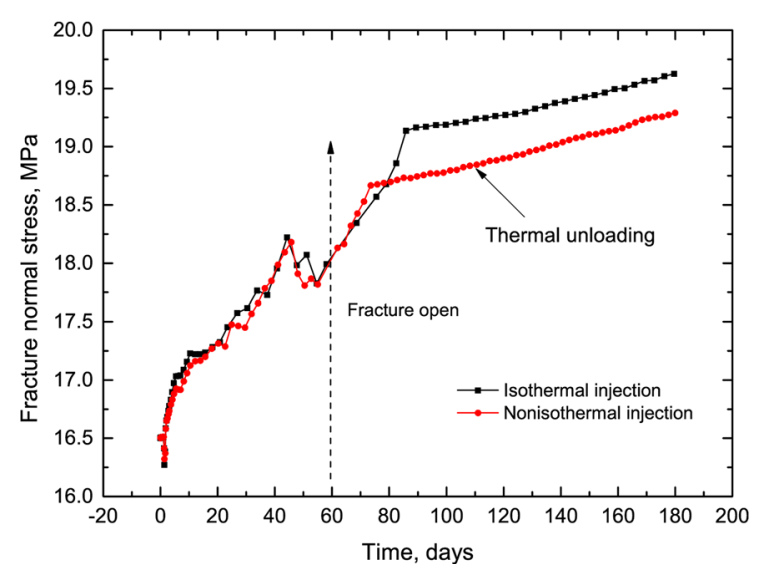

Fig. 10 Evolution of fracture normal stress under isothermal injection (black line) and non-isothermal injection (red line)

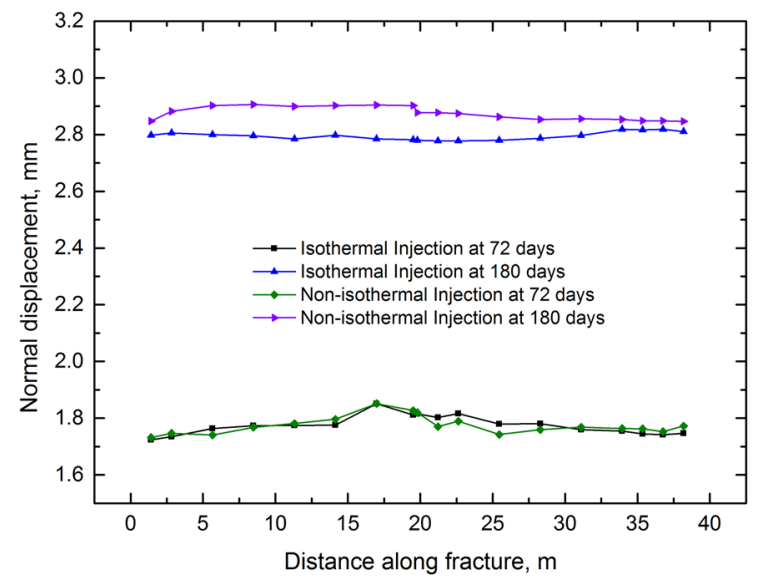

Fig. 11 Aperture distribution along the fracture at 72 and 180 days under conditions of both isothermal injection and nonisothermal injection

model TF_FLAC ${ }^{3 \mathrm{D}}$ and the original TFREACT (Fig. 13), which aims at validating the correctness of the developed equivalent continuum model in simulating the interaction of multi-fractures. In Fig. 12, there are two sets of orthogonal fractures (red and green lines) ubiquitously distributed in a $200 \mathrm{~m} \times 200 \mathrm{~m} \times 10 \mathrm{~m}$ reservoir. The fractures are separated at a constant spacing $4 \mathrm{~m}$. The injector is centrally located in the reservoir with a constant injection rate of $0.01 \mathrm{~m}^{3} / \mathrm{s}$. The reservoir properties are described in Table 1.

In the model of continuum TFREACT, the evolution of fracture permeability is represented by a hyperbolic function as, 
Fig. 12 Schematic of two sets of orthogonal fracture ubiquitously distributed in a $200 \mathrm{~m} \times 200 \mathrm{~m}$ reservoir

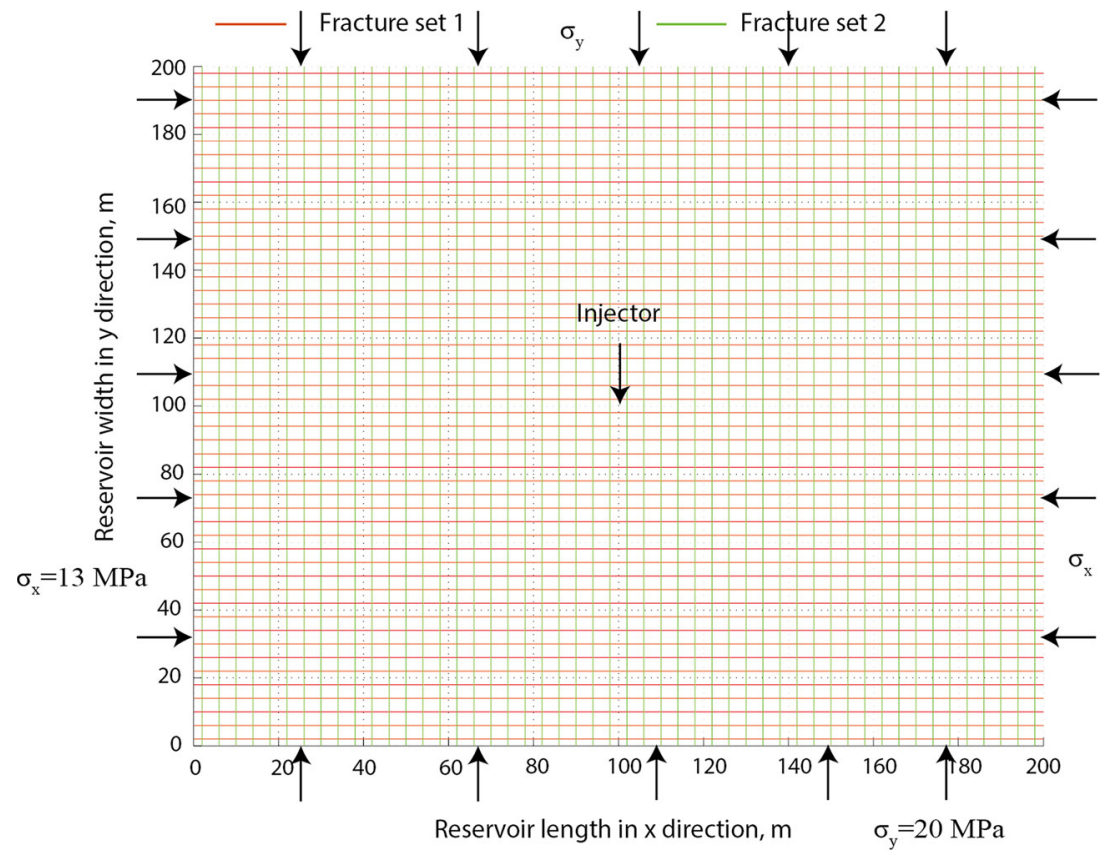

$b=b_{r}+\left(b_{m}-b_{r}\right) \times \exp \left(\sigma_{N} \times a\right)$

where $b_{r}$ is the residual aperture, $b_{m}$ is the maximum aperture, $\sigma_{N}$ is the effective normal stress of the fracture (MPa), $a$ is the non-linear fracture stiffness ( $1 /$ $\mathrm{MPa}$ ), which is defined as $1.4 \mathrm{MPa}^{-1}$ in this model. Figure 13 shows the evolution of fracture aperture over time. There is good agreement in the magnitude of fracture aperture between the continuum TFREACT and the equivalent continuum TF_FLAC ${ }^{3 \mathrm{D}}$. The injection pressure results are either validated in Fig. 14. When the fracture pressure increases, the decreased effective normal stress prompts the enhancement of fracture aperture.

\section{DFN application}

In realistic reservoir, fractures networks usually are non-uniformly distributed. Complex fracture pattern yields heterogeneous fluid flow and heat thermal drawdown (Bear 1993; Tsang and Neretnieks 1998), and the development of anisotropic fracture permeability, which could induce flow channeling in the major fractures (Chen et al. 1999; Min et al. 2004). In this section, a reservoir model with two discrete sets of fractures is constructed to address the evolution of

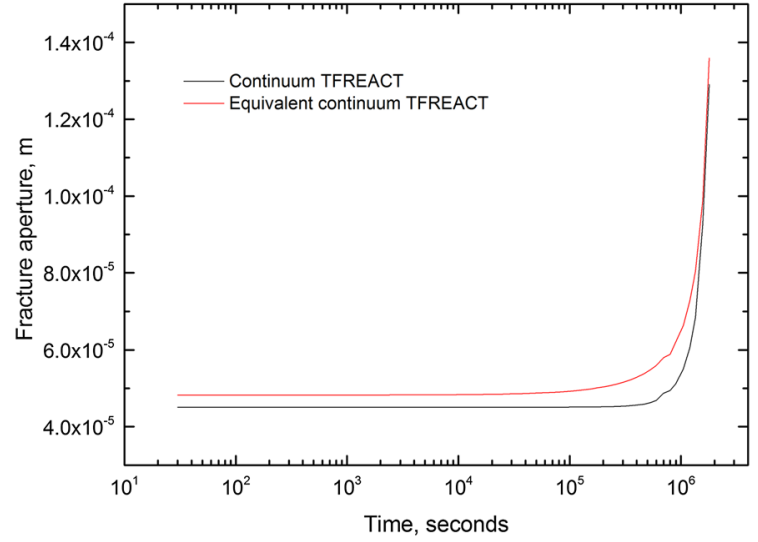

Fig. 13 Validation of fracture aperture at the injection well between the developed equivalent continuum TF_FLAC ${ }^{3 \mathrm{D}}$ and the original continuum TFREACT (Taron et al. 2009)

fracture permeability, due to the influence of stress state and fracture orientation.

\subsection{Fracture network generation}

To create the DFN in the simulation, various factors including fracture location, orientation, length, and aperture are necessarily considered. In this work, two sets of fractures are oriented at azimuths (from the North) of $020^{\circ}$ and $135^{\circ}$ in a $200 \mathrm{~m} \times 200 \mathrm{~m} \times 10 \mathrm{~m}$ 


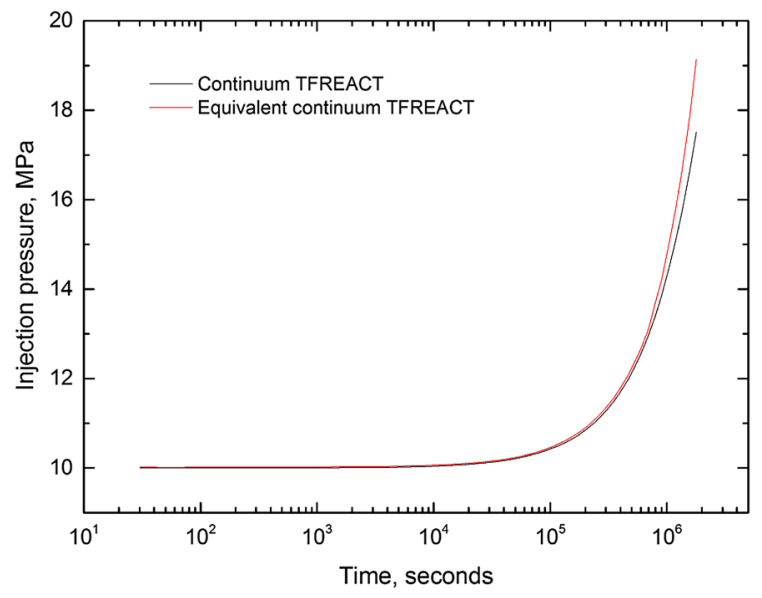

Fig. 14 Validation of injection pressure between the developed equivalent continuum TF_FLAC ${ }^{3 \mathrm{D}}$ and the original continuum TFREACT

reservoir (Fig. 15c). The injector is located at coordinate $(20,140)$. The geometry of each individual fracture is defined by prescribing the starting and ending point according to the fracture length and orientation (Fig. 15a). Each set of fractures comprises 50 individual fractures of various lengths distributed within the reservoir. There are several major fractures which allow the fluid to diffuse into the majority of reservoir. The length of fractures in the reservoir follows the traditional lognormal distribution (Fig. 15b) (de Dreuzy et al. 2001; Muralidharan et al. 2004). The fracture length is constrained in the range of 1-60 $\mathrm{m}$. The initial aperture for each fracture is determined by a power law function (Eq. 22) (Olson 2003), which implies that longer fractures of trace length $l$ have greater initial aperture $b_{i}$.

$b_{i}=1.25 \times 10^{-5} l^{0.8}$

Figure 16 indicates that the initial fracture aperture in this model varies from $5.0 \times 10^{-5} \mathrm{~m}$ to $2.5 \times 10^{-4} \mathrm{~m}$, corresponding to the predefined range of fracture length.

The reservoir properties in the DFN model are the same and are defined in Table 1 . In the base case, the maximum principal stress is $20 \mathrm{MPa}(\mathrm{N}-\mathrm{S}$ direction) and the minimum principal stress is $13 \mathrm{MPa}(\mathrm{E}-\mathrm{W}$ direction). The initial reservoir pressure is uniform at $10 \mathrm{MPa}$. The water is injected at a constant rate of $5.0 \times 10^{-6} \mathrm{~m}^{3} / \mathrm{s}$.
Figure 17a shows the resulting fracture pressure distribution at 180 days for the base case. The fracture permeability distribution in Fig. 17b corresponds to the shear stress drop along both fracture sets in Fig. 17c, d. The shear stress around the injector drops $\sim 3 \mathrm{MPa}$ for the fracture set oriented at $045^{\circ}$. Furthermore, the path of shear stress drop also follows with the path of major fractures, which increases the permeability of fractures.

\subsection{Effect of applied stress}

Applied boundary stress has a direct impact in determining the potential of fracture shear failure and the resulting permeability evolution. Therefore it is desirable to investigate the various ratios of boundary stress conditions influencing the evolution of fracture permeability. To evaluate the effect of stress state, two scenarios of the ratio of boundary stresses are proposed. Table 2 illustrates the composition of the sensitivity tests for these applied boundary stresses. The initial reservoir pressure and injection rate are kept constant for all the cases.

The first test category in Table 2 indicates the schedule for the constant stress ratio. The applied boundary stresses are increasing proportionally, such that the stress ratio of $\sigma_{x} / \sigma_{y}$ is kept constant at 0.65 . Figure 18 shows the fracture aperture distribution at 180 days for the two sets of fractures with increasing stress $\sigma_{x}$ from 13 to $39 \mathrm{MPa}$. When the applied stresses are proportionally increasing, the initial apertures for the both fracture sets decrease uniformly, the response of normal closure is the dominant behavior by decreasing the initial aperture, resulting from the increased fracture normal stress.

Figure 19 illustrates the evolution of fracture permeability close to injector under the fixed stress ratio. As the applied boundary stresses are augmenting proportionally, the failure potential declines. Fracture closure is the dominating response due to the increased normal stress, resulting in reduction of fracture permeability.

Conversely, the deviatoric stress is elevated in the second set of cases by increasing the boundary stress $\sigma_{x}$, while holding the applied boundary stress $\sigma_{y}$ constant at $20 \mathrm{MPa}$. In Fig. 20, the fracture permeability close to the injection well was selected to illustrate the development of permeability anisotropy 
Fig. 15 a Locations of starting points and ending points for the two sets of fractures in the reservoir, b lognormal probability distribution for the length of fractures, $\mathbf{c}$ distribution of fracture networks in a $200 \mathrm{~m} \times 200 \mathrm{~m} \times 10 \mathrm{~m}$ reservoir, fractures in red are oriented at $135^{\circ}$, and those in green are oriented at $020^{\circ}$ (with respect to the North)

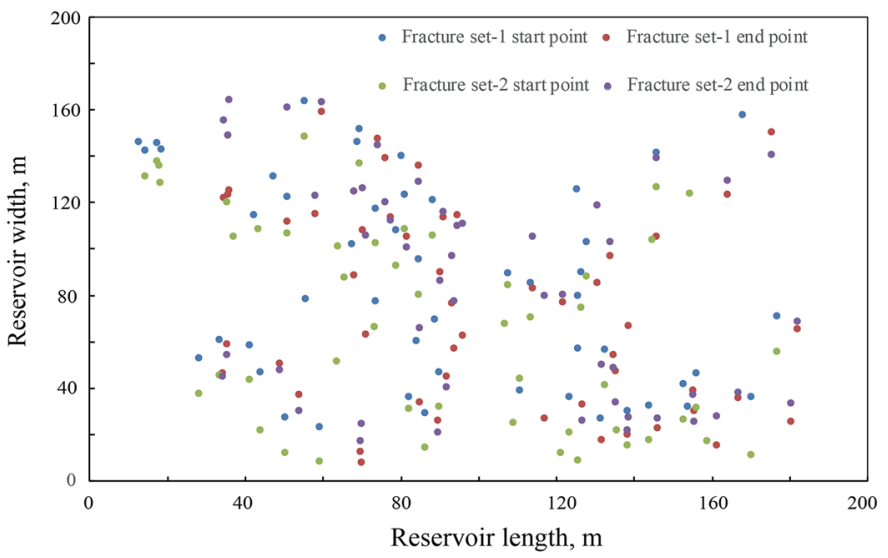

(a)

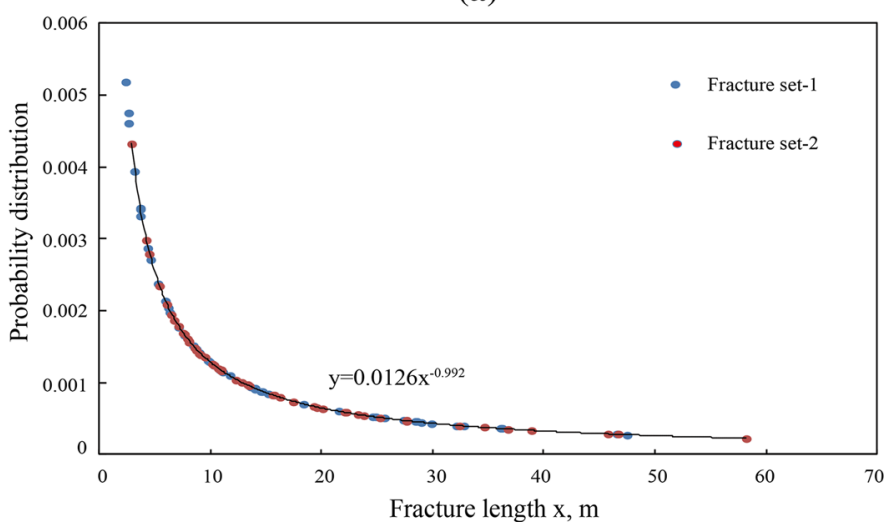

(b)
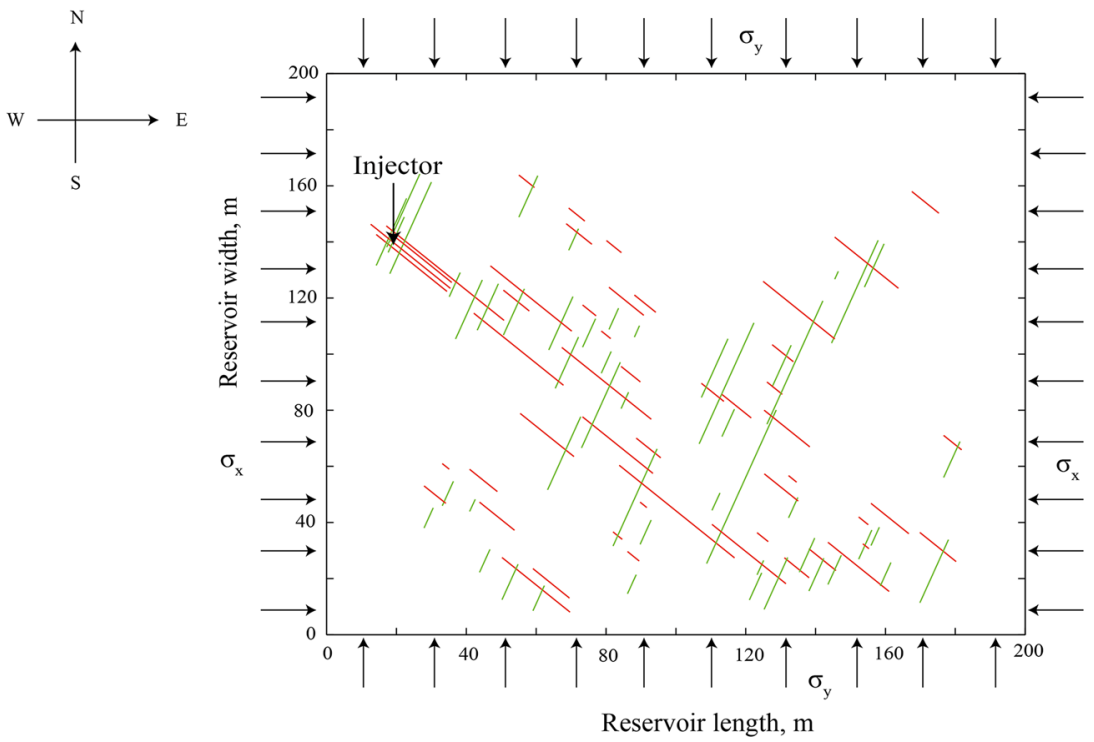

(c) when the stress difference is increasing. When the boundary stress $\sigma_{x}$ is increasing from 13 to $40 \mathrm{MPa}$, the anisotropy of fracture permeability emerges.
Figure 21 points out the fracture aperture evolutions for the two sets of fractures with an increasing deviatoric stress. As the applied boundary stress in 


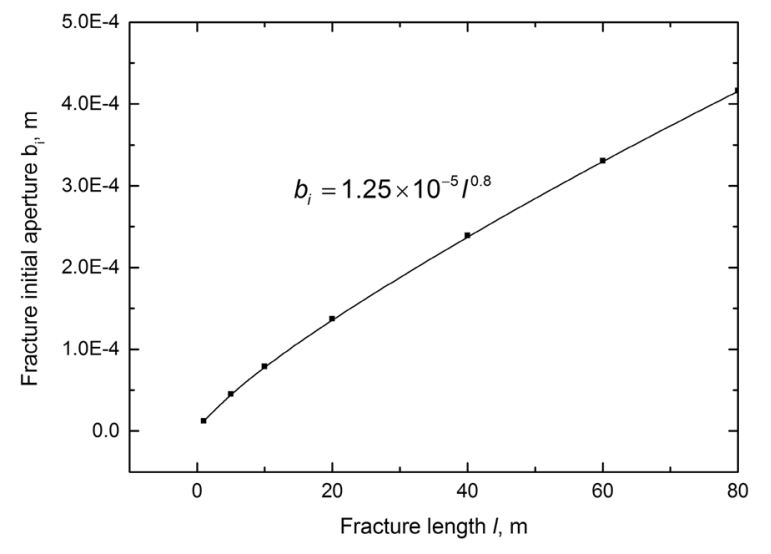

Fig. 16 Nonlinear power law relationship between the fracture length and fracture initial aperture (a)

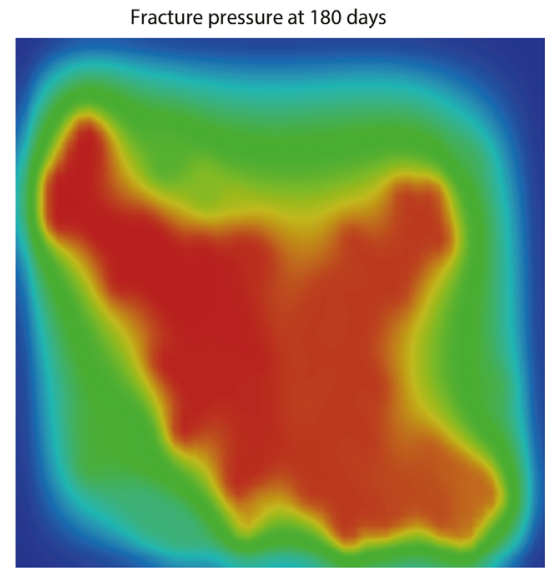

(c)

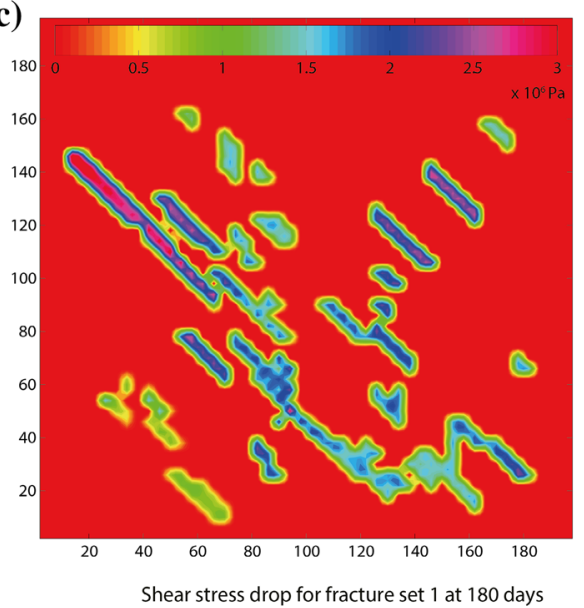

Table 2 Sensitivity tests of applied stress boundaries in the fracture aperture evolution

\begin{tabular}{llll}
\hline Test category & $\sigma_{x}(\mathrm{MPa})$ & $\sigma_{y}(\mathrm{MPa})$ & $\sigma_{x} / \sigma_{y}$ \\
\hline Stress ratio & 13 & 20 & 0.65 \\
& 19.5 & 30 & 0.65 \\
& 26 & 40 & 0.65 \\
Stress difference & 39 & 60 & 0.65 \\
& 30 & 20 & 1 \\
& 40 & 20 & 1.5 \\
& & 20 & 2 \\
\hline
\end{tabular}

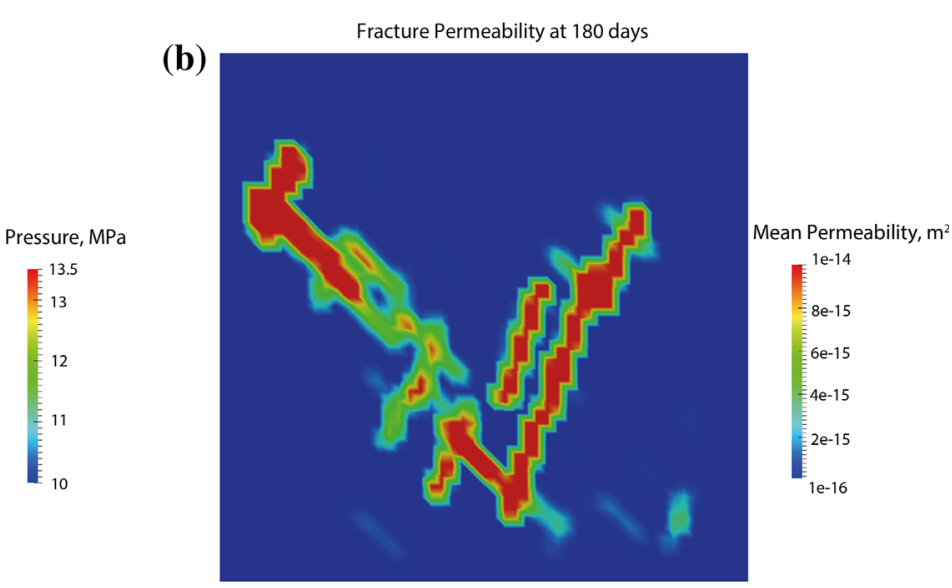

(d)

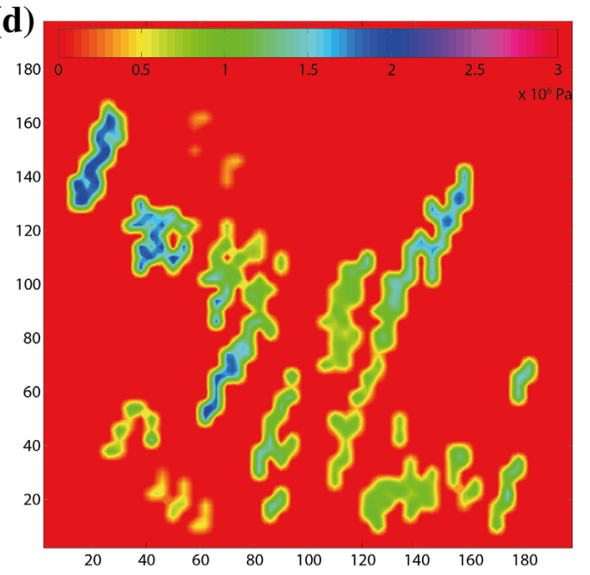

Shear stress drop for fracture set 2 at 180 days

Fig. 17 a Fracture pressure distribution for the base case at 180 days, $\mathbf{b}$ contour of fracture mean permeability, $\mathbf{c}$ shear stress drop along the first set of fractures, $\mathbf{d}$ shear stress drop along the second set of fractures 
Fig. 18 Fracture aperture distributions for the two sets of fractures under constant stress ratio at 180 days respectively
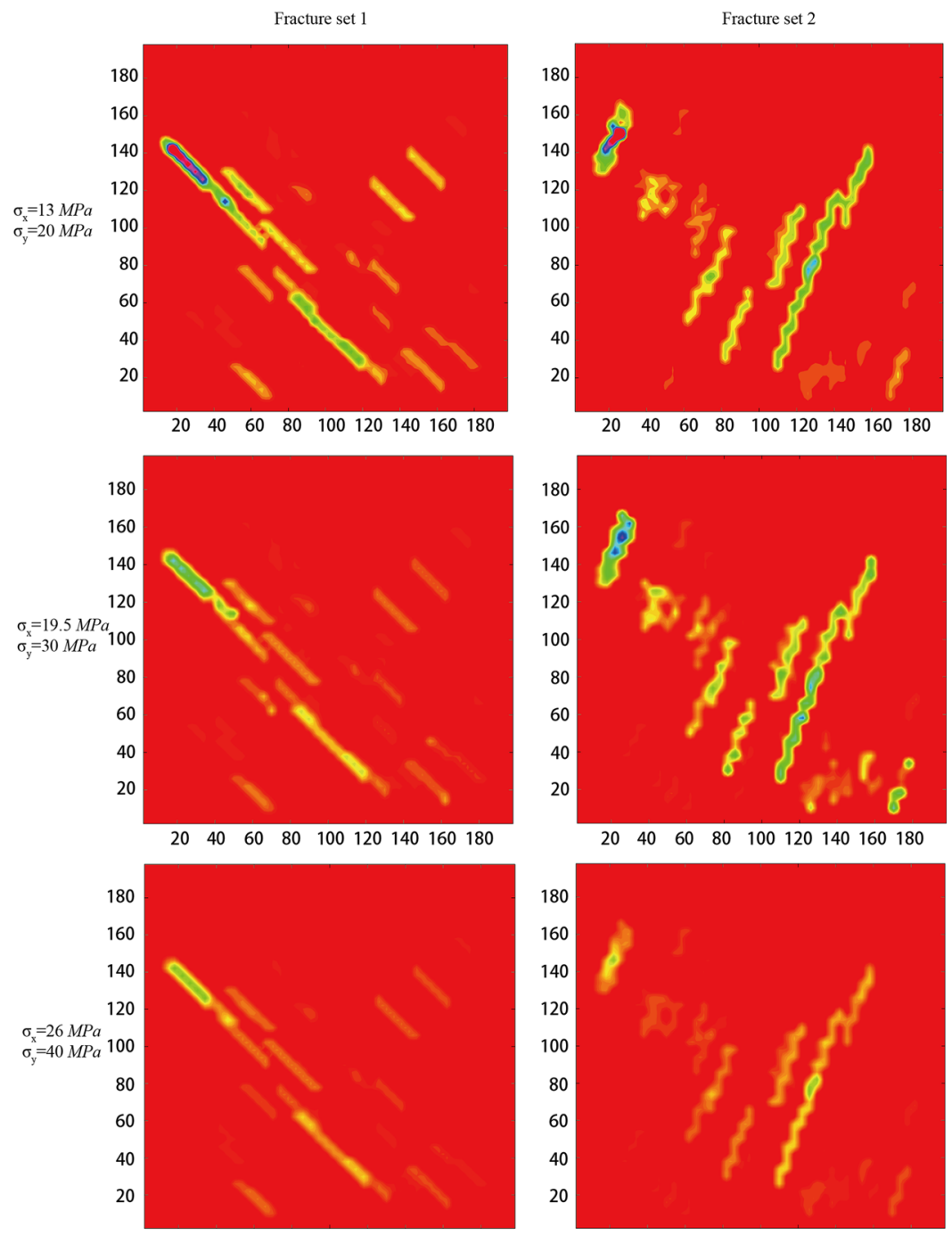

$\begin{array}{lllllllll}20 & 40 & 60 & 80 & 100 & 120 & 140 & 160 & 180\end{array}$

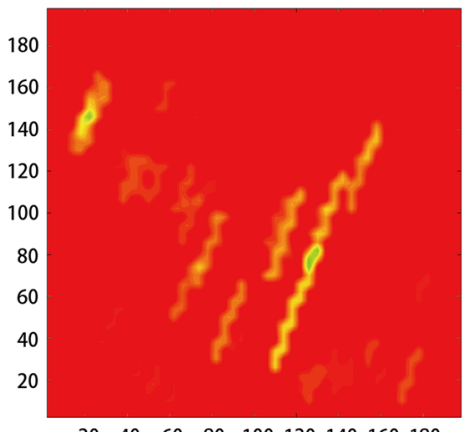

$\begin{array}{lllllllll}20 & 40 & 60 & 80 & 100 & 120 & 140 & 160 & 180\end{array}$

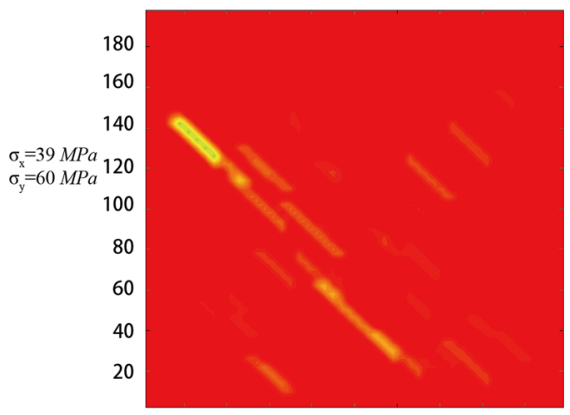

$\begin{array}{lllllllll}20 & 40 & 60 & 80 & 100 & 120 & 140 & 160 & 180\end{array}$

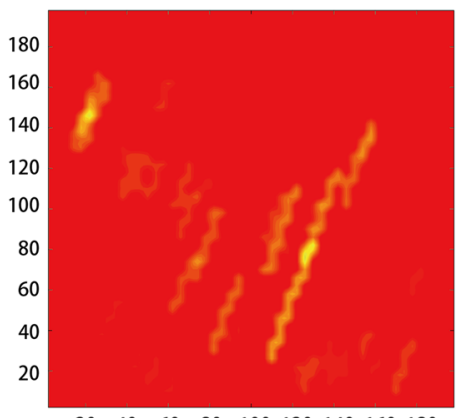

$20 \quad 40 \quad 60 \quad 80 \quad 100 \quad 120 \quad 140 \quad 160 \quad 180$

$\begin{array}{lllllllllll}0 & 0.5 & 1 & 1.5 & 2 & 2.5 & 3 & 3.5 & 4 & 4.5 & 5\end{array}$ 


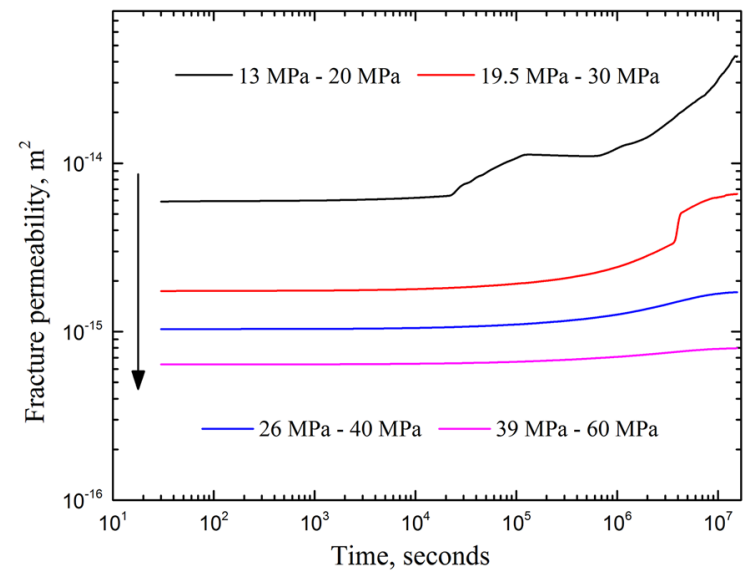

Fig. 19 Evolutions of fracture permeability around the injector under the fixed stress ratio within 180 days

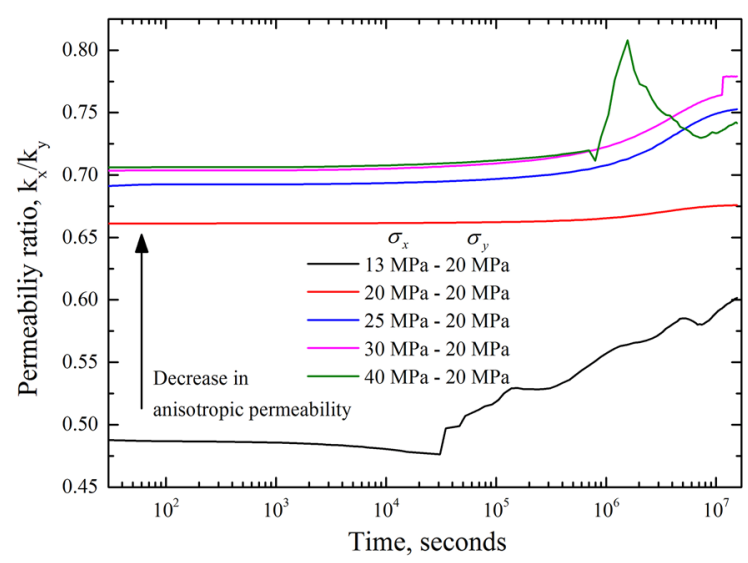

Fig. 20 Evolution of fracture permeability ratio $k_{x}$ over $k_{y}$ under different stress difference

the major principal stress direction (E-W direction) increases, the fractures normal to the major principal stress direction (fracture set 2) close, while the fractures aligned sub-parallel to the major principal stress (fracture set 1 ) are nearly critically stressed and have the greatest propensity to slip, dilate. Therefore the apertures in set 1 fractures are larger than those in set 2 fractures.

\subsection{Effect of fracture orientation}

Fracture orientation plays an important role in determining the stress state around the fracture, which will influence the potential of fracture failure and

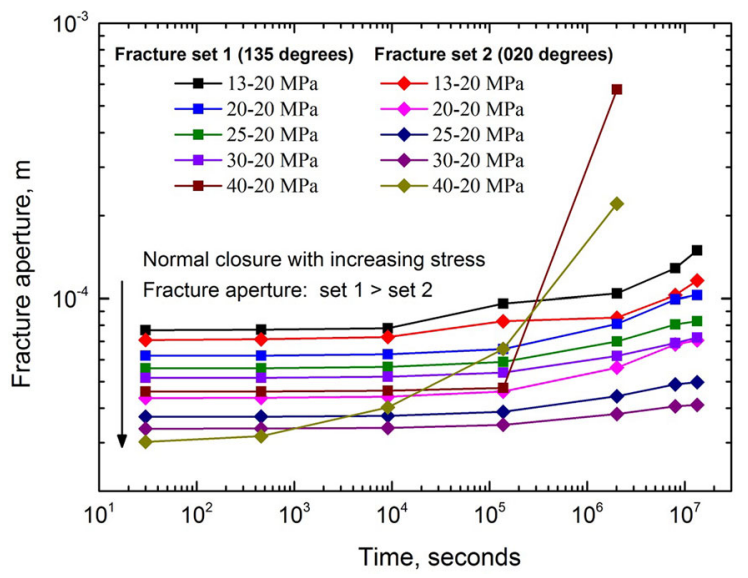

Fig. 21 Evolution of fracture aperture for the two sets of fracture with increasing stress difference. Normal closure is the dominating response as deviatoric stress increased. Fracture aperture in set 1 is larger than the set 2

permeability enhancement. In this study, the impact of fracture orientation is assessed by comparing the evolution of fracture permeability between the critically-stressed and non-critically stressed situations. Figure 22 shows the designated fracture network geometries striking at azimuths of $020^{\circ}-135^{\circ}$ (Fig. 22a), $060^{\circ}-120^{\circ}$ (Fig. 22b), and $015^{\circ}-165^{\circ}$ (Fig. 22c) (with respect to the North direction). The trace length for each fracture in the three cases is maintained the same magnitude, but at different azimuths. The network is not well connected when the set of fractures are oriented sub-parallel to the N-S direction (Fig. 22c). Figure 23 shows the distributions of fracture aperture at 180 days for different fracture orientations. Since the major principal stress is imposed in the $\mathrm{N}-\mathrm{S}$ direction, the acting normal stress on the fractures aligned sub-parallel to the $\mathrm{N}-\mathrm{S}$ direction (Fig. 23c) is smaller than the fractures oriented normal to the $\mathrm{N}-\mathrm{S}$ direction (Fig. 23b), therefore it can be seen that the fracture aperture around the injector with $015^{\circ}-165^{\circ}$ (Fig. 23c) are larger than the aperture in the case with $060^{\circ}-120^{\circ}$ (Fig. 23b). However, since the fractures are not well connected in the third case with $015^{\circ}-165^{\circ}$ orientation, the permeability enhancement is only focused around the injector zone, the fracture pressure build-up is high enough to trigger the local shear failure around the injection well. 

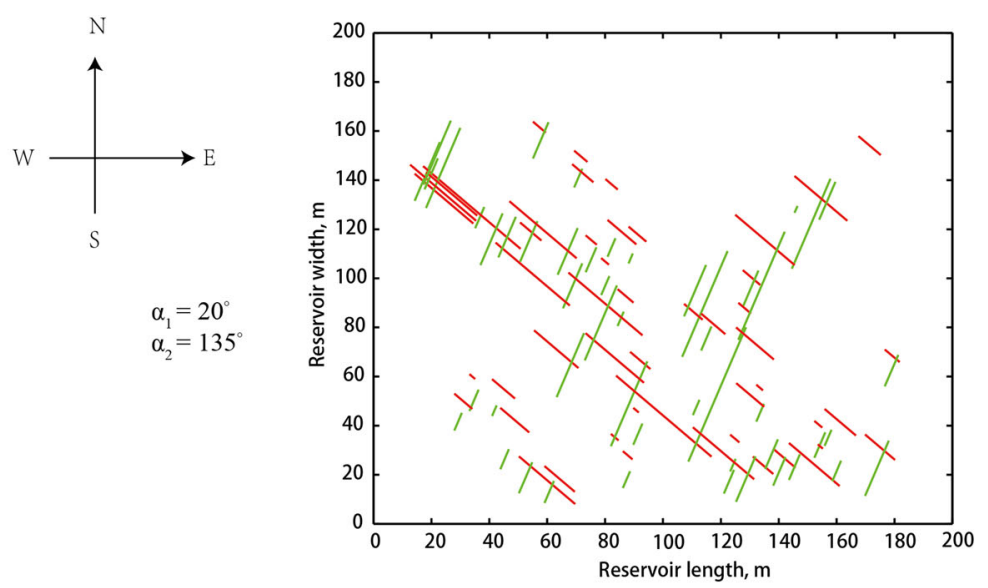

(a)

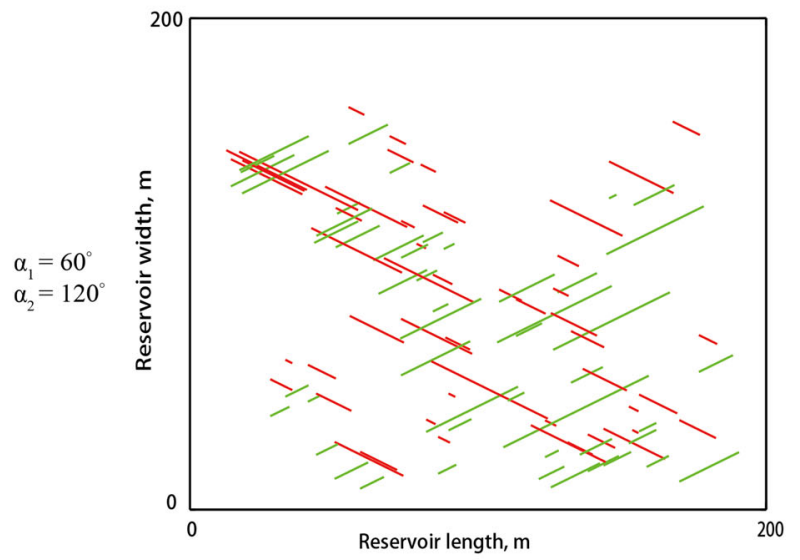

(b)

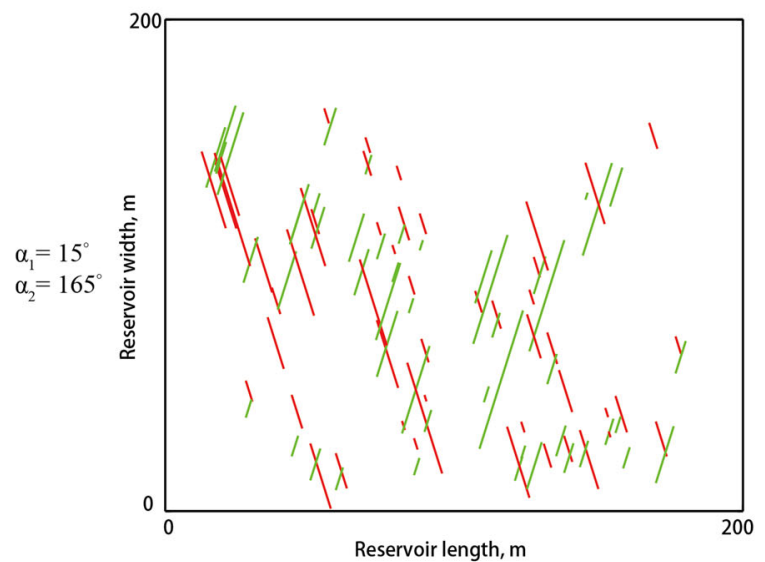

(c)

Fig. 22 Contour of fracture networks under different orientations representing the fractures oriented at $020^{\circ}-135^{\circ}, 060^{\circ}-120^{\circ}$, and $015^{\circ}-165^{\circ}$ with respect to the North separately

\section{Conclusion}

This work presents the development of an equivalent continuum model to represent randomly distributed fractured masses, and investigating the evolution of stress-dependent permeability of fractured rock masses. The model incorporates both mechanical crack tensor and permeability tensor approaches to characterize the pre-existing fractures, accommodating the orientation and trace length of fractures. Compared to other discrete fracture models, the advantages of this model are primarily represented in simulating the large scale reservoir including the long term coupled thermal-hydraulic-mechanical response, and also without any dependence in the fracture geometry and gridding. The accuracy of simulator has been evaluated against other discrete fracture models by examining the evolution of fracture aperture and injection pressure during injection. The simulator is shown capable of predicting the evolution of the fracture normal aperture, including the state of fracture normal closure, shear dilation, and out of contact displacements under tensile loading.

The influence of stress state (mean and deviatoric) and fracture orientation on the evolution of fracture permeability are assessed by applying the model to a DFN. Normal closure of the fracture system is the dominant mechanism in reducing fracture permeability, where the mean stress is augmented at a constant stress obliquity ratio of 0.65 . Conversely, for varied stress obliquity (0.65-2) shear deformation is the principal mechanism resulting in an increase in 

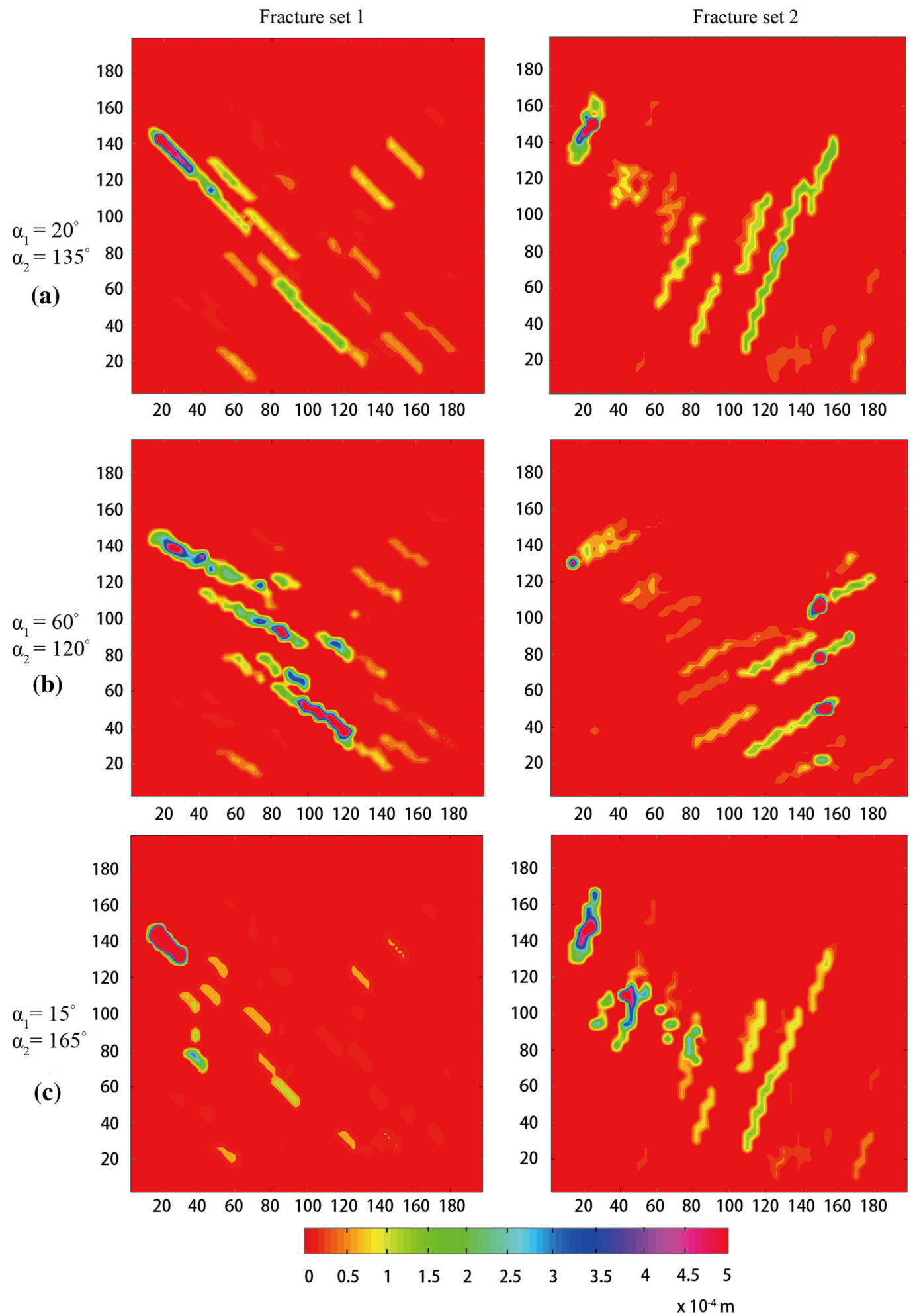

Fig. 23 Fracture aperture comparisons at 180 days between different fracture orientations at $020^{\circ}-135^{\circ}, 060^{\circ}-120^{\circ}$, and $015^{\circ}-165^{\circ}$ with respect to North 
permeability. Fractures aligned sub-parallel to the major principal stress are near critically-stressed and have the greatest propensity to slip, dilate and increase permeability. Those fractures normal to direction of the principal stress are subjected to the increasing normal stress, and reduce the permeability. These mechanisms increase the anisotropy of permeability in the rock mass. Furthermore, as the network becomes progressively more sparse, the loss of connectivity results in a reduction in permeability with zones of elevated pressure locked close to the injector-with the potential for elevated levels of induced seismicity.

Acknowledgments This work is a partial result of support from the US Department of Energy under project DOE-DE-343 EE0002761. This support is gratefully acknowledged. We also acknowledge the data from the University of Oklahoma and University of Texas at Austin.

Open Access This article is distributed under the terms of the Creative Commons Attribution 4.0 International License (http:// creativecommons.org/licenses/by/4.0/), which permits unrestricted use, distribution, and reproduction in any medium, provided you give appropriate credit to the original author(s) and the source, provide a link to the Creative Commons license, and indicate if changes were made.

\section{References}

Baghbanan A, Jing L (2007) Hydraulic properties of fractured rock masses with correlated fracture length and aperture. Int J Rock Mech Min Sci 44(5):704-719. doi:10.1016/j. ijrmms.2006.11.001

Bandis SC, Lumsden AC, Barton NR (1983) Fundamentals of rock joint deformation. Int J Rock Mech Min Sci Geomech Abstr 20(6):249-268. doi:10.1016/0148-9062(83)90595-8

Barton N, Choubey V (1977) The shear strength of rock joints in theory and practice. Rock Mech 10(1-2):1-54. doi:10. 1007/BF01261801

Bear J (1993) Modeling flow and contaminant transport in fractured rocks. In: de Marsily JB-FT (ed) Flow and contaminant transport in fractured rock. Academic Press, Oxford, pp 1-37. doi:10.1016/B978-0-12-083980-3.50005-X

Biot MA (1941) General theory of three-dimensional consolidation. J Appl Phys 12(2):155-164

Chen M, Bai M, Roegiers JC (1999) Permeability tensors of anisotropic fracture networks. Math Geol 31(4):335-373. doi:10.1023/A:1007534523363

Chin LY, Raghavan R, Thomas LK (2000) Fully coupled geomechanics and fluid-flow analysis of wells with stressdependent permeability. Soc Pet Eng. doi:10.2118/58968PA

Crouch SL, Starfield AM (1991) Bound elem solid mechanics. Taylor \& Francis, London de Dreuzy J-R, Davy P, Bour O (2001) Hydraulic properties of two-dimensional random fracture networks following a power law length distribution: 2. Permeability of networks based on lognormal distribution of apertures. Water Resour Res 37(8):2079-2095. doi:10.1029/2001WR900010

Dieterich JH (1992) Earthquake nucleation on faults with rateand state-dependent strength. Tectonophysics 211(1-4):115-134. doi:10.1016/0040-1951(92)90055-B

Elsworth D (1986) A hybrid boundary element-finite element analysis procedure for fluid flow simulation in fractured rock masses. Int $\mathrm{J}$ Numer Anal Methods Geomech 10(6):569-584. doi:10.1002/nag.1610100603

Fu P, Johnson SM, Carrigan CR (2013) An explicitly coupled hydro-geomechanical model for simulating hydraulic fracturing in arbitrary discrete fracture networks. Int $\mathbf{J}$ Numer Anal Methods Geomech 37(14):2278-2300. doi:10.1002/nag.2135

Gan Q, Elsworth D (2014a) Analysis of fluid injection-induced fault reactivation and seismic slip in geothermal reservoirs. J Geophys Res Solid Earth 119(4):3340-3353. doi:10. 1002/2013JB010679

Gan Q, Elsworth D (2014b) Thermal drawdown and late-stage seismic-slip fault reactivation in enhanced geothermal reservoirs. J Geophys Res Solid Earth 119(12):8936-8949. doi:10.1002/2014JB011323

Ghassemi A, Zhang Q (2006) Porothermoelastic analysis of the response of a stationary crack using the displacement discontinuity method. J Eng Mech 132(1):26-33. doi:10. 1061/(ASCE)0733-9399(2006)132:1(26)

Goodman RE, Taylor RL, Brekke TL (1968) A model for the mechanics of jointed rock. J Soil Mech Found Div Am Soc Civ Eng 94:637-660

Heuze FE, Shaffer RJ, Ingraffea AR, Nilson RH (1990) Propagation of fluid-driven fractures in jointed rock. Part $1-$ development and validation of methods of analysis. Int $\mathbf{J}$ Rock Mech Min Sci Geomech Abstr 27(4):243-254

Itasca Consulting Group, Inc. (2000) FLAC3D-fast Lagrangian analysis of continua in three-dimensions, ver. 5.0. Itasca, Minneapolis

Kelkar S, McClure M, Ghassemi A (2015) Influence of fracture shearing on fluid flow and thermal behavior of an EGS reservoir-geothermal code comparison study. Paper presented at fortieth workshop on geothermal reservoir engineering. Stanford University, Stanford, 26-28 Jan 2015

Kohl T, Evansi KF, Hopkirk RJ, Rybach L (1995) Coupled hydraulic, thermal and mechanical considerations for the simulation of hot dry rock reservoirs. Geothermics 24(3):345-359

McClure M, Horne RN (2013) Discrete fracture network modeling of hydraulic stimulation: coupling flow and geomechanics. Springer, Berlin

McTigue DF (1990) Flow to a heated borehole in porous, thermoelastic rock: analysis. Water Resour Res 26(8):1763-1774. doi:10.1029/WR026i008p01763

Min K-B, Jing L (2003) Numerical determination of the equivalent elastic compliance tensor for fractured rock masses using the distinct element method. Int J Rock Mech Min Sci 40(6):795-816. doi:10.1016/S1365-1609(03) 00038-8

Min K-B, Rutqvist J, Tsang C-F, Jing L (2004) Stress-dependent permeability of fractured rock masses: a numerical study. 
Int J Rock Mech Min Sci 41(7):1191-1210. doi:10.1016/j. ijrmms.2004.05.005

Muralidharan V, Chakravarthy D, Putra E, Schechter DS (2004) Investigating fracture aperture distributions under various stress conditions using X-ray CT scanner, edited. Petroleum Society of Canada. doi:10.2118/2004-230

Noorishad J, Ayatollahi MS, Witherspoon PA (1982) A finiteelement method for coupled stress and fluid flow analysis in fractured rock masses. Int J Rock Mech Min Sci Geomech Abstr 19(4):185-193. doi:10.1016/0148-9062(82) 90888-9

Oda M (1986) An equivalent continuum model for coupled stress and fluid flow analysis in jointed rock masses. Water Resour Res 22(13):1845-1856. doi:10.1029/ WR022i013p01845

Olson JE (2003) Sublinear scaling of fracture aperture versus length: an exception or the rule? J Geophys Res Solid Earth 108(B9):2413. doi:10.1029/2001JB000419

Pine RJ, Cundall PA (1985) Applications of the fluid-rock interaction program (FRIP) to the modelling of hot dry rock geothermal energy systems. In: Proceedings of international symposium on fundamentals of rock joints. Centek, pp 293-302
Rutqvist J, Stephansson O (2003) The role of hydromechanical coupling in fractured rock engineering. Hydrogeol J 11(1):7-40

Rutqvist J, Leung C, Hoch A, Wang Y, Wang Z (2013) Linked multicontinuum and crack tensor approach for modeling of coupled geomechanics, fluid flow and transport in fractured rock. J Rock Mech Geotech Eng 5(1):18-31. doi:10.1016/j. jrmge.2012.08.001

Taron J, Elsworth D (2010) Coupled mechanical and chemical processes in engineered geothermal reservoirs with dynamic permeability. Int $\mathrm{J}$ Rock Mech Min Sci 47(8):1339-1348. doi:10.1016/j.ijrmms.2010.08.021

Taron J, Elsworth D, Min K-B (2009) Numerical simulation of thermal-hydrologic-mechanical-chemical processes in deformable, fractured porous media. Int J Rock Mech Min Sci 46(5):842-854. doi:10.1016/j.ijrmms.2009.01.008

Tsang C-F, Neretnieks I (1998) Flow channeling in heterogeneous fractured rocks. Rev Geophys 36(2):275-298. doi:10.1029/97RG03319

Zhang X, Sanderson D (1994) Fractal structure and deformation of fractured rock masses. In: Kruhl $\mathrm{J}$ (ed) Fractals and dynamic systems in geoscience. Springer, Berlin, pp 37-52. doi:10.1007/978-3-662-07304-9_3 